\title{
Assessment of recent evidence for the management of patients with systemic sclerosis-associated interstitial lung disease: a systematic review
}

\author{
Anna-Maria Hoffmann-Vold ${ }^{1,7}$, Toby M. Maher ${ }^{2,3,7}$, Edward E. Philpot ${ }^{4}$, \\ Ali Ashrafzadeh ${ }^{5}$ and Oliver Distler ${ }^{6}$
}

Affiliations: ${ }^{1}$ Dept of Rheumatology, Oslo University Hospital-Rikshospitalet, Oslo, Norway. ${ }^{2}$ National Institute of Health Research Respiratory Clinical Research Facility, Royal Brompton Hospital, London, UK. ${ }^{3}$ Fibrosis Research Group, National Heart and Lung Institute, Imperial College London, London, UK. ${ }^{4}$ Respiratory Center of Excellence, IQVIA, Durham, NC, USA. ${ }^{5}$ Rheumatology Center of Excellence, IQVIA, San Diego, CA USA. ${ }^{6}$ Dept of Rheumatology, University Hospital Zurich, Zurich, Switzerland. ${ }^{7}$ These authors contributed equally.

Correspondence: Anna-Maria Hoffmann-Vold, Dept of Rheumatology, Oslo University Hospital, PO boks 4950 Nydalen, 0424 Oslo, Norway. E-mail: a.m.hoffmann-voldamedisin.uio.no

ABSTRACT This systematic review summarises current evidence to help guide treatment decisions for patients with systemic sclerosis (SSc)-associated interstitial lung disease (ILD). A systematic search of the literature (January 2012 to April 2018), including grey literature (searched between 1992 and 2011), was conducted using multiple electronic databases. Guidelines, meta-analyses, randomised controlled trials and observational studies reporting on risk stratification, screening, diagnosis, treatment and management outcomes for patients with SSc-ILD were included. A quality assessment of the included evidence was undertaken.

In total, 2464 publications were identified and 280 included. Multiple independent risk factors for ILD in patients with SSc were identified, including older age, male sex and baseline pulmonary function. Highresolution computed tomography (HRCT) has been used for characterising ILD in patients with SSc, and pulmonary function tests are a key adjunctive component in the diagnostic and monitoring pathway. The clinical value of biomarkers relating to SSc-ILD diagnosis or assessment for disease progression is unknown at present. Immunosuppressive therapy (monotherapy or combined therapy) is the current standard of care for SSc-ILD; long-term evidence for effective and safe treatment of SSc-ILD is limited.

Identification of patients at risk for SSc-ILD remains challenging. HRCT and pulmonary function tests are key to diagnosing and monitoring for disease progression. Although immunosuppressive therapy is considered current first-line treatment, it is partly associated with adverse effects and long-term follow-up evidence is limited. Novel therapies and biomarkers should be further explored in well-controlled clinical studies.

@ERSpublications

This systematic literature review on systemic sclerosis-associated interstitial lung disease summarises the evidence supporting approaches to disease management in clinical practice https://bit.ly/2ISdSNc

Cite this article as: Hoffmann-Vold A-M, Maher TM, Philpot EE, et al. Assessment of recent evidence for the management of patients with systemic sclerosis-associated interstitial lung disease: a systematic review. ERJ Open Res 2021; 7: 00235-2020 [https://doi.org/10.1183/23120541.00235-2020].

This article has supplementary material available from openres.ersjournals.com

Received: 1 May 2020 | Accepted: 9 Dec 2020

Copyright $\odot$ ERS 2021. This article is open access and distributed under the terms of the Creative Commons Attribution Licence 4.0. 


\section{Introduction}

Systemic sclerosis (SSc) is a rare, heterogeneous autoimmune disease characterised by immune-mediated inflammatory processes, vasculopathy and fibrosis, which is clinically manifested by multiorgan involvement $[1,2]$. Although the aetiology of SSc is unknown, evidence suggests links between genetic predisposition and environmental factors [3]. Interstitial lung disease (ILD) is a frequent organ manifestation and is a leading cause of morbidity and mortality in patients with SSc $[1,4]$. As such, early identification of patients who are at risk of organ disease as well as the subsequent monitoring of these patients are of importance in improving clinical outcomes $[5,6]$.

The early identification of SSc-ILD is challenging, as symptoms are usually subclinical. Consequently, SSc-ILD is frequently diagnosed during advanced stages of disease. There is a paucity of evidence-based guidelines for the screening and early diagnosis of SSc-ILD, as well as limited scientific information that may be used to guide treatment decisions [7]. Long-term, effective treatment options for SSc-ILD are scarce and current treatment approaches focus on targeting inflammatory pathways with immunosuppressive therapy $[8,9]$. In daily clinical practice, treatment initiation is often based on the presence of factors that are related to either expected ongoing lung disease progression or to baseline disease severity.

The objective of this systematic review is to summarise the available scientific literature to help guide decisions for screening, management and monitoring disease progression for patients diagnosed with SSc-ILD. These results formed the basis for the development of evidence-based consensus statements on the identification and management of SSc-ILD [10].

\section{Methods}

Search strategy

The search strategy methods are described in the supplementary methods.

\section{Outcomes}

Data were collected for the following key outcomes: risk factors for SSc-ILD (comorbidities, biomarkers, polymorphisms); screening (tests for currently undiagnosed disease), diagnosis (tests used to diagnose SSc-ILD) and assessment of disease severity; treatment initiation and options; and disease progression (tests and markers for disease progression).

\section{Results}

A total of 2464 citations were retrieved, of which 1894 unique abstracts were identified. Screening of these abstracts led to the inclusion of 708 publications for full-text review, out of which 447 publications were ineligible. In addition, the grey literature search using Google Scholar yielded a total of 360 articles, of which 19 publications were relevant. A total of 280 publications (244 original studies; 36 publications associated with an original study) were considered eligible for inclusion in the review (figure 1). Most of the evidence is based on observational studies $(n=237 ; 85 \%)$; six (2.1\%) were randomised controlled trials (RCTs).

\section{Quality of the evidence}

The overall risk of bias was low among the six included RCTs. Details on the statistical analysis plan and allocation concealment were missing from most of the studies. One open-label RCT was only published as an abstract and not available as a full paper at the time of analysis (table 1) [14].

The quality of the 235 publications from observational studies was judged to be high for the reporting of primary outcomes ( $92 \%$ of the studies). $98 \%$ of these studies included quantitative analyses. There was adequate reporting of treatment exposure in $75 \%$ of the studies. However, the quality of publications was judged to be low for reporting and handling of confounding factors and immortal time bias (the period of follow-up time during which the outcome of interest cannot occur), and validation of study primary outcomes, with $<50 \%$ of publications reporting these details. Almost one-third $(30 \%)$ of the included observational studies were reported as conference abstracts only (supplementary table S2).

\section{Risk factors}

The evidence was assessed for risk factors that may be associated with the presence, severity and progression of SSc-ILD.

High-quality evidence reported an association between diffuse skin involvement and ILD [17-22]. Moderate-quality evidence suggested that antibody status of anti-centromere (ACA) and anti-topoisomerase I (ATA) are risk factors associated with ILD [18, 19], with ACA being protective for SSc-ILD and ATA increasing the likelihood of the presence of SSc-ILD [18, 23, 24]. Other evidence identified additional risk factors for the presence of SSc-ILD, including older age, male sex [19, 20] and 
FIGURE 1 Selection process of included publications (Preferred Reporting Items for Systematic Reviews and Meta-Analyses diagram).

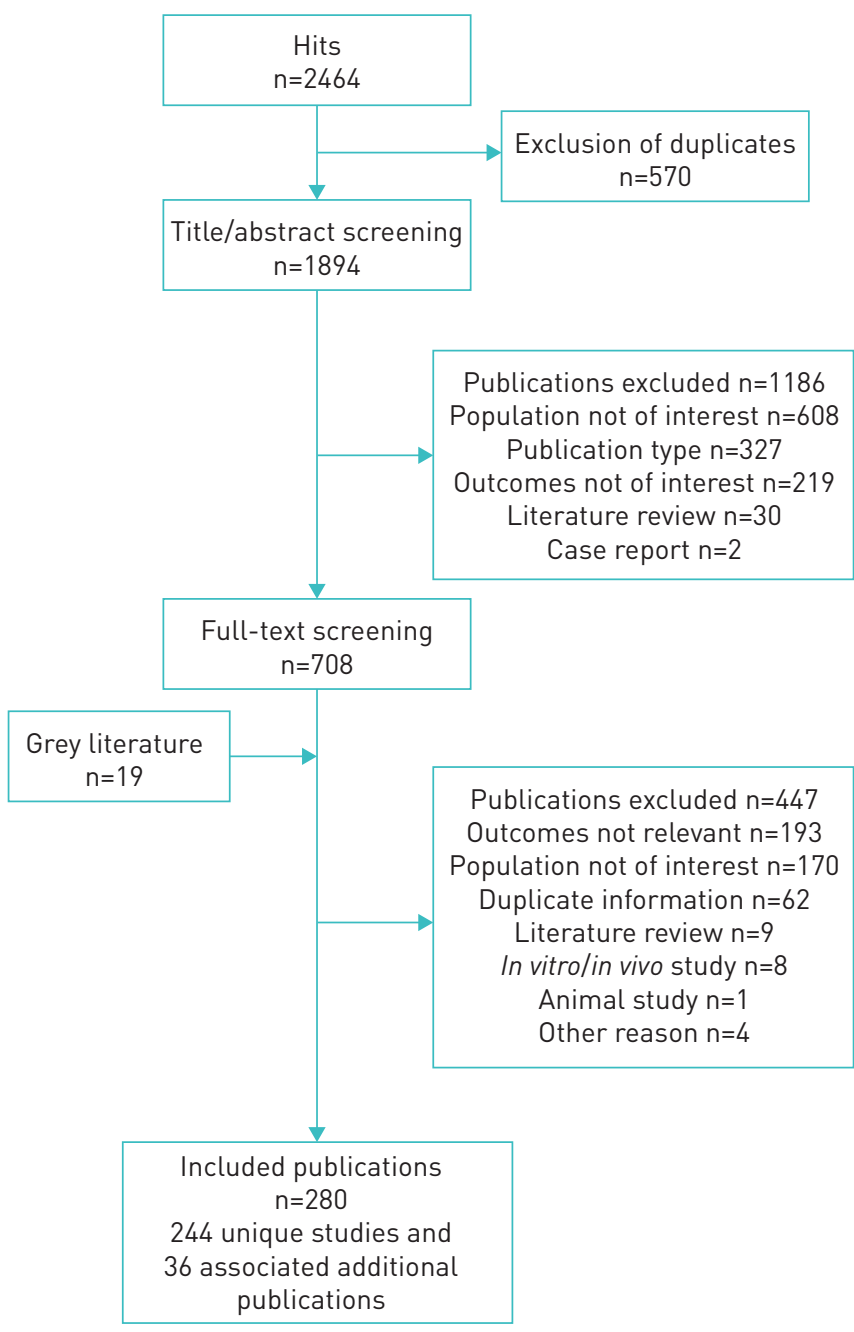

baseline forced vital capacity (FVC) and diffusing capacity of the lung for carbon monoxide $\left(D_{\mathrm{LCO}}\right)$ [25, 26]. High-quality evidence reported that high-resolution computed tomography (HRCT) findings can be used to assess the severity of SSc-ILD at baseline $[18,27,28]$. Baseline C-reactive protein levels correlated with long-term decline in FVC in patients with early SSc [29].

Supplementary table S3 provides a comprehensive list of other risk factors associated with SSc-ILD.

Screening, diagnosis and assessment of disease severity

Moderate-quality evidence suggests that pulmonary function tests (including FVC and $D_{\mathrm{LCO}}$ ) in combination with HRCT may be useful in screening for SSc-ILD [25, 30, 31]. No data were reported for the optimal timing for screening.

\section{TABLE 1 Critical appraisal of randomised controlled trials using National Institute for Health and Care Excellence standards ${ }^{\#}$}

First author, year [ref.]

TASHKIN, 2007 [12]

TASHKIN, 2016 [13]

SIRCAR, 2017" [14]

Pérez Campos, 2012 [15]

Seibold, 2010 [16]

\section{Randomisation}

Allocation concealment

Blinding

Follow-up

Selective reporting

Statistical analysis plan

\#: a further study was underpowered and therefore not rated at the same evidence level [11]; ": please note that since the date of the literature search, this reference (abstract) has been published in full. 
HRCT was identified as the most common method for diagnosing SSc-ILD (supplementary table S3). Moderate-quality evidence identified lung ultrasound as a potential tool to detect the presence of ILD in patients with SSc $[32,33]$.

Other evidence reported on other tests, including prognostic non-HRCT-based algorithms, serial cardiopulmonary exercise tests, nailfold capillaroscopy and other less commonly used tests that have been used for detecting ILD in SSc patients (supplementary table S3).

In the Scleroderma Lung Study (SLS) I, the extent of ILD defined by HRCT was a predictor of decline in FVC [34]. Moreover, pulmonary function tests (PFTs) including FVC and $D_{\text {LCO }}$ have been used as surrogate measures to assess the presence and severity of SSc-ILD at baseline (moderate-quality evidence) $[26,35]$. Moderate-quality evidence found that frequent cough correlated with the presence and severity of SSc-ILD [36].

\section{Treatment and options (initiation, escalation, rescue)}

Immunosuppressive drugs (monotherapy and combination) were predominantly used (supplementary table S3). No evidence or recommendations were identified for when and how to escalate treatment doses.

There is overall high-quality evidence supporting cyclophosphamide (CYC) [11, 12, 37]. SLS I [12] reported that treatment with CYC improved lung function (FVC \% predicted, total lung capacity \% predicted) compared with placebo in patients with SSc-ILD at 1 year. Another trial showed a trend for increased efficacy of low-dose prednisolone and intravenous CYC followed by oral azathioprine compared with placebo in an underpowered study [11].

There is also moderate evidence supporting treatment with mycophenolate mofetil (MMF) [13, 38]. SLS II [13] found that treatment with CYC for 1 year or MMF for 2 years both resulted in significant improvements in pre-specified measures of lung function (FVC \% predicted), lung imaging, dyspnoea and skin disease. MMF was more tolerable and less toxic, yet this study failed its primary end-point of superiority of 2 years' treatment with MMF over 1 year's treatment with CYC, and other RCTs do not exist for MMF. These data support the effectiveness of both treatments for progressive SSc-ILD and the current preference for MMF because it is more tolerable and associated with fewer adverse effects [13].

Haematopoietic stem cell transplantation should be considered for the treatment of carefully selected patients with rapidly progressing SSc who are at risk of organ failure. Improvements in FVC were seen and sustained at 2-year follow-up [39, 40]; however, the adverse event rate reported in one study was $43 \%$ and included two (14\%) patients with severe cardiomyopathy, of which one case was fatal (high-quality evidence) [41].

Moderate-quality evidence supports rituximab as a potential therapy in SSc-ILD [35, 42, 43].

Moderate-quality evidence indicates that lung transplantation is a valid treatment for highly selected ILD or pulmonary arterial hypertension patients with SSc [44].

High-quality evidence for the inefficacy of bosentan was identified [16]. References for studies on other therapies can be found in supplementary table S3.

Nonpharmacological treatments were not assessed.

\section{Disease progression}

PFTs and HRCT were identified as the most common measures to monitor the disease in SSc-ILD patients (supplementary table S3).

High-quality evidence identified HRCT as a useful imaging tool to determine the disease pattern in patients with SSc-ILD, correlating with PFTs $[25,31,45,46]$. High-quality evidence indicates that baseline HRCT can predict survival [47].

High-quality evidence found that disease progression, defined as either FVC decline from baseline $\geqslant 10 \%$ or FVC decline of $5-9 \%$ with a $D_{\text {LCO }}$ decline of $\geqslant 15 \%$, was associated with increased risk of mortality [48]. In a study investigating predictors of mortality in patients in SLS I and II, an FVC decline over 2 years was a superior predictor of mortality compared with baseline FVC [49].

Other evidence identified decline in exercise-induced blood oxygen saturation and arthritis as predictors of ILD progression in patients with SSc who had mild ILD [50].

A correlation between frequent cough and the presence or severity of SSc-ILD has been identified and is recognised as a symptom of disease progression in patients with SSc-ILD [36, 51]. 
Additional evidence was found for measures associated with disease progression, including exhaled nitric oxide, oesophageal diameter, pulmonary artery/ascending aorta ratio, arthritis and FVC values within the first 3 years following diagnosis (supplementary table S3).

\section{Circulating biomarkers}

The evidence was assessed for biomarkers that may be diagnostic for the presence or severity of SSc-ILD or prognostic for disease progression. Moderate-quality evidence found that surfactant protein-D serum levels correlated with markers of ILD severity, lung function and lung fibrosis in patients with SSc-ILD [52-54]. High CC chemokine ligand 18 (CCL18) levels were associated with disease progression and were predictive of lower survival rates and deterioration of pulmonary function [55]. However, in a different study, CCL18 levels were only predictive of short-term decline in FVC in patients with early SSc [53]. C-X-C motif chemokine ligand 4 levels were linked to progression of lung fibrosis [56] and Krebs von den Lungen-6 may be a useful biomarker of disease severity in SSc-ILD [57].

A list of other biomarkers identified in the systematic review can be found in supplementary table S3.

\section{Discussion}

A comprehensive systematic literature review was conducted and provides a broad summary on the SSc-ILD literature landscape regarding screening, diagnosis, management and disease progression. This review provides the foundation for the evidence-based consensus statements for the identification and management of SSc-ILD [10]. A total of 280 publications (244 original studies; 36 publications associated with an original study) were considered eligible for inclusion in the review. $85 \%$ of the evidence is based on observational studies.

Screening patients with SSc for lung disease is important for identifying patients with SSc-ILD early. In one study, $30 \%$ of patients with SSc-ILD showed progressive disease, defined by PFT decline, which was associated with decreased survival compared with stable FVC [4]. Progressive ILD is associated with poorer outcomes [4] and therefore regular monitoring of all patients with SSc-ILD is needed to identify those with progression and facilitate treatment decisions [58]. However, of key concern in patients with SSc-ILD are the complex and diverse clinical features that make early identification difficult [59]. The methods with highest-quality evidence identified to screen, diagnose and monitor the disease in SSc-ILD patients were PFTs and HRCT, which are routinely used in clinical practice. HRCT can detect ILD even when PFTs are normal [30], and chest HRCT using limited CT slices can even detect mild SSc-ILD while reducing patient exposure to radiation [60]. Several biomarkers, with varying levels of sensitivity and specificity, have also been investigated within clinical studies for the purpose of diagnosis, severity assessment and prediction of progression [23, 24, 52-55, 61-63]. However, the potential application of these biomarkers in the real-world hospital setting is unclear and needs further confirmation with more clinical evidence. The evidence may be indicative of potentially useful markers in the future.

Treatment of SSc-ILD is also challenging because of the clinical complexity and heterogeneity of the disease. Lung function decline has been shown to occur early in the disease course, and early treatment initiation may improve patient outcomes [64]. However, more recent evidence shows that patients may have a slower course of disease progression, demonstrating the importance of monitoring to identify patients at risk of progression [65].

Several different treatment approaches and strategies for SSc-ILD have been published recently [2, 66, 67]. Our systematic literature review showed that immunosuppressive therapies are the current standard of care in the treatment of SSc-ILD. CYC has been used frequently in the treatment of SSc-ILD, especially as induction therapy. Potential drug toxicity makes it unsuitable for long-term use. Intravenous administration has been shown to be preferable because it is less toxic than oral CYC [11]. MMF is widely suggested as an alternative immunosuppressive therapy for induction and maintenance and has been shown to stabilise lung function, but it actually lacks highest-level evidence, as primary end-point positive RCTs are missing. Our findings for CYC concur with recommendations included in the European League Against Rheumatism recommendations for treatment of patients with SSc who have lung disease [7]. To date, no randomised placebo-controlled trials investigating rituximab in SSc-ILD have been conducted. Observational and noncontrolled studies have shown that rituximab may benefit patients with SSc-ILD by preventing lung function decline and thus may be a potential future treatment when further RCT studies can prove efficacy $[42,43,68,69]$. Haematopoietic stem cell transplantation is a potential therapy for certain groups of patients with SSc, although adverse events are frequent, including treatment-related mortality [39-41].

Other immunosuppressive treatments are frequently used in clinical practice, but the evidence base supporting their use is not well described [70]. Thus, the availability of effective treatment options for 
patients with SSc-ILD remains limited. After our literature search, additional data were published that were used in the development of the evidence-based consensus statements. This included results from the SENSCIS trial showing that nintedanib is an effective treatment for patients with SSc-ILD [71]. Nintedanib is now approved for the treatment of patients with SSc-ILD in the United States and in Europe [72, 73]. Some important RCTs relating to other therapeutic agents have been published since the systematic literature review [2]. Data from the phase II faSScinate and phase III focuSSced studies investigating tocilizumab as a treatment for early, inflammatory diffuse cutaneous SSc indicate that it has a beneficial effect on ILD disease progression as measured by FVC decline and HRCT, despite the focuSSced study not meeting its primary end-point (the modified Rodnan skin score) [74-76]. On the basis of these studies, both nintedanib and tocilizumab were considered in the consensus statements [10].

The efficacy of treatments in SSc-ILD has been reviewed in detail elsewhere $[2,66]$. Nonpharmacological treatments and management strategies such as supplemental oxygen, pulmonary rehabilitation, physical activity and encouraging smoking cessation $[66,77]$ may have a role in supporting patients with SSc-ILD, but were not evaluated in this systematic review.

This systematic literature review has some limitations. Full publication of abstracts that were identified could have taken place since the search date in April 2018; this may impact on the current quality assessment of some of the included studies. There are no quality assessments existing that are universally applicable to cover the wide range of prediction, assessment and management covered in this systematic literature review, and thus compromises had to be made. The Good Research for Comparative Effectiveness checklist [78] does not have an option for "not applicable", and therefore a lower quality indicator was applied if information was not available, for example from an abstract, which reduced the overall quality of some studies, particularly if they were not published as a full article or if they were not clinical trials. This was often the case for noninterventional studies. In addition, there is the potential for subjectivity by the steering committee in assigning overall quality ratings to the evidence.

A strength of this study was that a comprehensive search of the literature was conducted, using multiple electronic sources and grey literature to identify the current evidence on risk stratification, screening, diagnosis, monitoring, treatment (patterns, pathways, escalation, algorithm) and ongoing management (including criteria for disease progression and biomarkers) outcomes for patients with SSc-ILD. A standardised methodology was used to minimise researcher bias. This review has informed the development of Delphi-based consensus statements for screening, diagnosis, treatment and assessment of disease progression in SSc-ILD, and to develop a management algorithm that will provide a framework for future decision-making in SSc-ILD [10].

\section{Conclusion}

SSc is a complex disease with diverse manifestations, which makes identification of clinically relevant ILD frequently challenging. Current evidence supports the notion that HRCT is a robust tool to diagnose, determine extent of disease and to identify disease progression of SSc-ILD. More research is required on the optimal quantification method and alternative imaging techniques. Several biomarkers have been investigated, but their relevance in clinical practice needs confirmation. This systematic review has highlighted the lack of high-quality evidence for guidance or recommendations for treatment algorithms and choice of treatments for patients with SSc-ILD. There is a need for further robust clinical research to evaluate alternative safe and effective treatment options for patients with SSc-ILD.

Acknowledgements: The authors meet criteria for authorship as recommended by the International Committee of Medical Journal Editors. The authors did not receive payment for the development of the review. The systematic literature review was conducted independently by IQVIA. Medical writing, editorial support and formatting assistance was provided by Julie Brown (AMICULUM Ltd, Auckland, New Zealand), and Claire Scott (MediTech Media, London, UK), which was contracted and funded by Boehringer Ingelheim International GmbH (BI). BI was given the opportunity to review the manuscript for medical and scientific accuracy as well as intellectual property considerations.

Conflict of interest: A-M. Hoffmann-Vold had a consultancy relationship and/or has received research funding from Actelion, Bayer, Boehringer Ingelheim, GlaxoSmithKline and Roche. T.M. Maher has, via his institution, received industry-academic funding from GlaxoSmithKline R\&D and UCB and has received consultancy or speakers' fees from Apellis, Astra Zeneca, Bayer, Blade Therapeutics, Boehringer Ingelheim, Bristol-Myers Squibb, Galapagos, GlaxoSmithKline R\&D, Indalo, Novartis, Pliant, ProMetic, Respivnat, Roche, Samumed and UCB. E.E. Philpot was an employee of IQVIA at the time of the study. At the time of the study, A. Ashrafzadeh was an employee of IQVIA, which has been supported by Boehringer Ingelheim for this study. O. Distler reports grants and personal fees from Actelion, personal fees from Abbvie, Acceleron Pharma, Anamar and Amgen, grants and personal fees from Bayer and Boehringer Ingelheim, personal fees from Catenion, CSL Behring, Ergonex, GSK, Inventiva, Italfarmaco, IQVIA, Medac and Medscape, grants and personal fees from Mitsubishi, personal fees from MSD, Lilly and Novartis, personal fees and nonfinancial support from Pfizer, personal fees from Roche, Target BioScience, UCB, Baecon Discovery, Blade Therapeutics, Curzion Pharmaceuticals, Glenmark Pharmaceuticals, Competitive Drug Development International Ltd and Galapagos NV, outside the submitted work; in addition, he has a patent (US8247389, EP2331143) issued. 
Support statement: Funding for the systematic literature review and medical writing support was provided by Boehringer Ingelheim. Funding information for this article has been deposited with the Crossref Funder Registry.

\section{References}

1 Schoenfeld SR, Castelino FV. Interstitial lung disease in scleroderma. Rheum Dis Clin North Am 2015; 41: 237-248.

2 Distler O, Volkmann ER, Hoffmann-Vold AM, et al. Current and future perspectives on management of systemic sclerosis-associated interstitial lung disease. Expert Rev Clin Immunol 2019; 15: 1009-1017.

3 Varga J, Trojanowska M, Kuwana M. Pathogenesis of systemic sclerosis: recent insights of molecular and cellular mechanisms and therapeutic opportunities. J Scleroderma Relat Disord 2017; 2: 137-152.

4 Hoffmann-Vold AM, Fretheim H, Halse AK, et al. Tracking impact of interstitial lung disease in systemic sclerosis in a complete nationwide cohort. Am J Respir Crit Care Med 2019; 200: 1258-1266.

5 Hoffmann-Vold AM, Distler O, Murray B, et al. Setting the international standard for longitudinal follow-up of patients with systemic sclerosis: a Delphi-based expert consensus on core clinical features. RMD Open 2019; 5: e000826.

6 Jaeger VK, Wirz EG, Allanore Y, et al. Incidences and risk factors of organ manifestations in the early course of systemic sclerosis: a longitudinal EUSTAR study. PLoS One 2016; 11: e0163894.

7 Kowal-Bielecka O, Fransen J, Avouac J, et al. Update of EULAR recommendations for the treatment of systemic sclerosis. Ann Rheum Dis 2017; 76: 1327-1339.

8 Suliman S, Al Harash A, Roberts WN, et al. Scleroderma-related interstitial lung disease. Respir Med Case Rep 2017; 22: 109-112

9 Mango RL, Ryu JH, Makol A. Newer insights into the management of interstitial lung disease in systemic sclerosis. Indian J Rheumatol 2017; 12: 194-203.

10 Hoffmann-Vold A-M, Maher TM, Philpot EE, et al. The identification and management of interstitial lung disease in systemic sclerosis: evidence-based European consensus statements. Lancet Rheumatol 2020; 2: e71-e83.

11 Hoyles RK, Ellis RW, Wellsbury J, et al. A multicenter, prospective, randomized, double-blind, placebo-controlled trial of corticosteroids and intravenous cyclophosphamide followed by oral azathioprine for the treatment of pulmonary fibrosis in scleroderma. Arthritis Rheum 2006; 54: 3962-3970.

12 Tashkin DP, Elashoff R, Clements PJ, et al. Effects of 1-year treatment with cyclophosphamide on outcomes at 2 years in scleroderma lung disease. Am J Respir Crit Care Med 2007; 176: 1026-1034.

13 Tashkin DP, Roth MD, Clements PJ, et al. Mycophenolate mofetil versus oral cyclophosphamide in scleroderma-related interstitial lung disease (SLS II): a randomised controlled, double-blind, parallel group trial. Lancet Respir Med 2016; 4: 708-719.

14 Sircar G, Goswami RP, Rath D, et al. A randomized controlled, open label trial of cyclophosphamide versus rituximab in diffuse systemic sclerosis. Indian J Rheumatol 2017; 12: Suppl. 1, OC022.

15 Pérez Campos D, Estévez Del Toro M, Peña Casanovas A, et al. Are high doses of prednisone necessary for treatment of interstitial lung disease in systemic sclerosis? Reumatol Clin 2012; 8: 58-62.

16 Seibold JR, Denton CP, Furst DE, et al. Randomized, prospective, placebo-controlled trial of bosentan in interstitial lung disease secondary to systemic sclerosis. Arthritis Rheum 2010; 62: 2101-2108.

17 Hafez EA, Hamza SH, Morad CS, et al. Pulmonary manifestations in Egyptian patients with systemic sclerosis. Egypt Rheumatol 2018; 40: 39-44.

18 Sánchez-Cano D, Ortego-Centeno N, Callejas J, et al. Interstitial lung disease in systemic sclerosis: data from the Spanish Scleroderma Study Group. Rheumatol Int 2018; 38: 363-374.

19 Wangkaew S, Euathrongchit J, Wattanawittawas P, et al. Incidence and predictors of interstitial lung disease (ILD) in Thai patients with early systemic sclerosis: inception cohort study. Mod Rheumatol 2016; 26: 588-593.

20 Gonçalves DR, Fonseca R, Aguiar F, et al. Determinants associated with interstitial pulmonar involvement in patients with systemic sclerosis - a cross-sectional study. Ann Rheum Dis 2016; 75: Suppl. 2, 748.

21 Ashmore $\mathrm{P}$, Tikly M, Wong M, et al. Interstitial lung disease in South Africans with systemic sclerosis. Rheumatol Int 2018; 38: 657-662.

22 Zamora FD, Kim HJ, Wang Q. Prevalence of pulmonary function test abnormalities and their correlation to high resolution computer tomography in a large scleroderma population. Am J Respir Crit Care Med 2013; 187: A2920.

23 Arandia NI, Simeón-Aznar CP, Castillo MD, et al. Influence of antibody profile in clinical features and prognosis in a cohort of Spanish patients with systemic sclerosis. Clin Exp Rheumatol 2017; 35: S98-S105.

24 Liaskos C, Marou E, Simopoulou T, et al. Disease-related autoantibody profile in patients with systemic sclerosis. Autoimmunity 2017; 50: 414-421.

25 Yap V, Zantah M, Athwal P, et al. Correlation of PFT parameters with HRCT-fibrosis score in scleroderma patients. Chest 2016; 150: 476A.

26 Le Gouellec N, Duhamel A, Perez T, et al. Predictors of lung function test severity and outcome in systemic sclerosis-associated interstitial lung disease. PLoS One 2017; 12: e0181692.

27 Martyanov V, Kim GJ, Hayes W, et al. Novel lung imaging biomarkers and skin gene expression subsetting in dasatinib treatment of systemic sclerosis-associated interstitial lung disease. PLoS One 2017; 12: e0187580.

28 Tashkin D, Volkmann ER, Tseng C-H, et al. Relationship between quantitative radiographic assessments of interstitial lung disease and physiological and clinical features of systemic sclerosis. Ann Rheum Dis 2016; 75: 374-381.

29 Liu X, Mayes MD, Pedroza C, et al. Does C-reactive protein predict the long-term progression of interstitial lung disease and survival in patients with early systemic sclerosis? Arthritis Care Res 2013; 65: 1375-1380.

30 Showalter K, Hoffmann A, Rouleau G, et al. Performance of forced vital capacity and lung diffusion cutpoints for associated radiographic interstitial lung disease in systemic sclerosis. J Rheumatol 2018; 45: 1572-1576.

31 Wangkaew S, Euathrongchit J, Wattanawittawas P, et al. Correlation of delta high-resolution computed tomography (HRCT) score with delta clinical variables in early systemic sclerosis (SSc) patients. Quant Imaging Med Surg 2016; 6: 381-390.

32 Gigante A, Rossi Fanelli F, Lucci S, et al. Lung ultrasound in systemic sclerosis: correlation with high-resolution computed tomography, pulmonary function tests and clinical variables of disease. Intern Emerg Med 2016; 11: 213-217. 
33 Song G, Bae SC, Lee YH. Diagnostic accuracy of lung ultrasound for interstitial lung disease in patients with connective tissue diseases: a meta-analysis. Clin Exp Rheumatol 2016; 34: 11-16.

34 Khanna D, Nagaraja V, Tseng C-H, et al. Predictors of lung function decline in scleroderma-related interstitial lung disease based on high-resolution computed tomography: implications for cohort enrichment in systemic sclerosis-associated interstitial lung disease trials. Arthritis Res Ther 2015; 17: 372.

35 Daoussis D, Melissaropoulos K, Sakellaropoulos G, et al. A multicenter, open-label, comparative study of B-cell depletion therapy with rituximab for systemic sclerosis-associated interstitial lung disease. Semin Arthritis Rheum 2017; 46: 625-631.

36 Tashkin DP, Volkmann ER, Tseng C-H, et al. Improved cough and cough-specific quality of life in patients treated for scleroderma-related interstitial lung disease: results of Scleroderma Lung Study II. Chest 2017; 151: 813-820.

37 Barnes H, Holland AE, Westall GP, et al. Cyclophosphamide for connective tissue disease-associated interstitial lung disease. Cochrane Database Syst Rev 2018; 1: CD010908.

38 Tzouvelekis A, Galanopoulos N, Bouros E, et al. Effect and safety of mycophenolate mofetil or sodium in systemic sclerosis-associated interstitial lung disease: a meta-analysis. Pulm Med 2012; 2012: 143637.

39 Burt RK, Shah SJ, Dill K, et al. Autologous non-myeloablative haemopoietic stem-cell transplantation compared with pulse cyclophosphamide once per month for systemic sclerosis (ASSIST): an open-label, randomised phase 2 trial. Lancet 2011; 378: 498-506.

40 van Laar JM, Farge D, Sont JK, et al. Autologous hematopoietic stem cell transplantation vs intravenous pulse cyclophosphamide in diffuse cutaneous systemic sclerosis: a randomized clinical trial. JAMA 2014; 311: 2490-2498.

41 Nakamura H, Odani T, Yasuda S, et al. Autologous haematopoietic stem cell transplantation for Japanese patients with systemic sclerosis: long-term follow-up on a phase II trial and treatment-related fatal cardiomyopathy. Mod Rheumatol 2018; 25: 879-884

42 Sari A, Guven D, Armagan B, et al. Rituximab experience in patients with long-standing systemic sclerosis-associated interstitial lung disease: a series of 14 patients. J Clin Rheumatol 2017; 23: 411-415.

43 Lepri G, Avouac J, Airò P, et al. Effects of rituximab in connective tissue disorders related interstitial lung disease. Clin Exp Rheumatol 2016; 34: 181-185.

44 Fernández-Codina A, Berastegui C, Pinal-Fernández I, et al. Lung transplantation in systemic sclerosis: a single center cohort study. Joint Bone Spine 2018; 85: 79-84.

45 Bosello S, Occhipinti ME, Canestrari G, et al. Quantitative CT evaluation in diffuse interstitial lung involvement in systemic sclerosis: usefulness of lung texture analysis to predict the functional change over time. Arthritis Rheumatol 2017; 69: Suppl. 10, 742.

46 Goldin JG, Kim GHJ, Kleerup E, et al. Association of changes in quantitative CT with outcome measures in the Scleroderma Lung Study II. Am J Respir Crit Care Med 2017; 195: A7416.

47 Takei R, Arita M, Kumagai S, et al. Radiographic fibrosis score predicts survival in systemic sclerosis-associated interstitial lung disease. Respirology 2018; 23: 385-391.

48 Goh NS, Hoyles RK, Denton CP, et al. Short-term pulmonary function trends are predictive of mortality in interstitial lung disease associated with systemic sclerosis. Arthritis Rheumatol 2017; 69: 1670-1678.

49 Volkmann ER, Tashkin DP, Sim M, et al. The course of the forced vital capacity during treatment for systemic sclerosis-related interstitial lung disease predicts long-term survival in 2 independent cohorts. Arthritis Rheumatol 2017; 69: Suppl. 10, 943.

$50 \mathrm{Wu} \mathrm{W}$, Jordan S, Becker MO, et al. Prediction of progression of interstitial lung disease in patients with systemic sclerosis: the SPAR model. Ann Rheum Dis 2018; 77: 1326-1332.

51 Theodore AC, Tseng $\mathrm{C}-\mathrm{H}, \mathrm{Li} \mathrm{N}$, et al. Correlation of cough with disease activity and treatment with cyclophosphamide in scleroderma interstitial lung disease: findings from the Scleroderma Lung Study. Chest 2012 142: 614-621.

52 Volkmann ER, Tashkin DP, Hant FN, et al. Surfactant protein D and Krebs von den Lungen-6 predict severity of systemic sclerosis-related interstitial lung disease in two independent cohorts. Arthritis Rheumatol 2016; 68: Suppl. 10,3245 .

53 Elhaj M, Charles J, Pedroza C, et al. Can serum surfactant protein D or CC-chemokine ligand 18 predict outcome of interstitial lung disease in patients with early systemic sclerosis? J Rheumatol 2013; 40: 1114-1120.

54 Yamakawa H, Hagiwara E, Kitamura H, et al. Serum KL-6 and surfactant protein-D as monitoring and predictive markers of interstitial lung disease in patients with systemic sclerosis and mixed connective tissue disease. J Thorac Dis 2017; 9: 362-371.

55 Hoffmann-Vold AM, Tennøe AH, Garen T, et al. High level of chemokine CCL18 is associated with pulmonary function deterioration, lung fibrosis progression, and reduced survival in systemic sclerosis. Chest 2016; 150: 299-306.

56 Volkmann ER, Tashkin DP, Roth MD, et al. Changes in plasma CXCL4 levels are associated with improvements in lung function in patients receiving immunosuppressive therapy for systemic sclerosis-related interstitial lung disease. Arthritis Res Ther 2016; 18: 305.

57 Kennedy B, Branagan P, Moloney F, et al. Biomarkers to identify ILD and predict lung function decline in scleroderma lung disease or idiopathic pulmonary fibrosis. Sarcoidosis Vasc Diffuse Lung Dis 2015; 32: 228-236.

58 Wijsenbeek M, Kreuter M, Olson A, et al. Progressive fibrosing interstitial lung diseases: current practice in diagnosis and management. Curr Med Res Opin 2019; 35: 2015-2024.

59 Scholand MB, Carr E, Frech T, et al. Interstitial lung disease in systemic sclerosis: diagnosis and management. Rheumatology 2012; Suppl. 1, 8 .

60 Frauenfelder T, Winklehner A, Nguyen TD, et al. Screening for interstitial lung disease in systemic sclerosis: performance of high-resolution CT with limited number of slices: a prospective study. Ann Rheum Dis 2014; 73: 2069-2073.

61 Kranenburg P, van den Hombergh WM, Knaapen-Hans HK, et al. Survival and organ involvement in patients with limited cutaneous systemic sclerosis and anti-topoisomerase-I antibodies: determined by skin subtype or auto-antibody subtype? A long-term follow-up study. Rheumatology 2016; 55: 2001-2008.

62 Caetano J, Nihtyanova S, Harvey J, et al. Distinctive clinical phenotype of anti-centromere antibody-positive diffuse systemic sclerosis. Arthritis Rheumatol 2016; 68: 111-112. 
van Bon L, Affandi AJ, Broen J, et al. Proteome-wide analysis and CXCL4 as a biomarker in systemic sclerosis. $N$ Engl J Med 2014; 370: 433-443.

64 Steen VD, Medsger TA Jr. Severe organ involvement in systemic sclerosis with diffuse scleroderma. Arthritis Rheum 2000; 43: 2437-2444.

65 Hoffmann-Vold A-M, Allanore Y, Alves M, et al. Progressive interstitial lung disease in patients with systemic sclerosis-associated interstitial lung disease in the EUSTAR database. Ann Rheum Dis 2021; 80: $219-227$.

66 Hoffmann-Vold AM, Allanore Y, Bendstrup E, et al. The need for a holistic approach for SSc-ILD - achievements and ambiguity in a devastating disease. Respir Res 2020; 21: 197.

67 Roofeh D, Jaafar S, Vummidi D, et al. Management of systemic sclerosis-associated interstitial lung disease. Curr Opin Rheumatol 2019; 31: 241-249.

68 Daoussis D, Liossis SC, Tsamandas AC, et al. Effect of long-term treatment with rituximab on pulmonary function and skin fibrosis in patients with diffuse systemic sclerosis. Clin Exp Rheumatol 2012; 30: S17-S22.

69 Jordan S, Distler JH, Maurer B, et al. Effects and safety of rituximab in systemic sclerosis: an analysis from the European Scleroderma Trial and Research (EUSTAR) group. Ann Rheum Dis 2015; 74: 1188-1194.

70 Adler S, Huscher D, Siegert E, et al. Systemic sclerosis associated interstitial lung disease - individualized immunosuppressive therapy and course of lung function: results of the EUSTAR group. Arthritis Res Ther 2018; 20: 17.

71 Distler O, Highland KB, Gahlemann M, et al. Nintedanib for systemic sclerosis-associated interstitial lung disease. N Engl J Med 2019; 380: 2518-2528.

72 U.S. Food and Drug Administration. OFEV (nintedanib): Prescribing Information. www.accessdata.fda.gov/ drugsatfda_docs/label/2020/205832s013lbl.pdf Date last updated: March 2020. Date last accessed: September 24 2020.

73 European Medicines Agency. OFEV ${ }^{\oplus}$ (nintedanib): Summary of Product Characteristics. www.ema.europa.eu/en/ documents/product-information/ofev-epar-product-information_en.pdf Date last updated: July 28, 2020. Date last accessed: September 24, 2020.

74 Khanna D, Denton CP, Lin CJF, et al. Safety and efficacy of subcutaneous tocilizumab in systemic sclerosis: results from the open-label period of a phase II randomised controlled trial (faSScinate). Ann Rheum Dis 2018; 77: $212-220$.

75 Khanna D, Lin CJF, Goldin J, et al. OP0245 Preservation of lung function observed in a phase 3 randomized controlled trial of tocilizumab for the treatment of early SSc. Ann Rheum Dis 2019; 78: 202-203.

76 Khanna D, Lin CJF, Furst DE, et al. Tocilizumab in systemic sclerosis: a randomised, double-blind, placebo-controlled, phase 3 trial. Lancet Respir Med 2020; 8: 963-974.

77 de Oliveira NC, Portes LA, Pettersson H, et al. Aerobic and resistance exercise in systemic sclerosis: state of the art. Musculoskeletal Care 2017; 15: 316-323.

78 Dreyer NA, Velentgas P, Westrich K, et al. The GRACE checklist for rating the quality of observational studies of comparative effectiveness: a tale of hope and caution. J Manag Care Spec Pharm 2014; 20: 301-308. 